\title{
Imaging Evaluation of Male Breast Masses with Histopathologic Correlation: A Case Series
}

\author{
Tanvi P. Vaidya ${ }^{1}$ Subhash K. Ramani ${ }^{2}$ \\ ${ }^{1}$ Department of Radiology, Ruby Hall Clinic, Pune, Maharashtra, \\ India \\ 2Department of Radiology, D Y Patil Hospital, Navi Mumbai, \\ Maharashtra, India
}

\author{
Address for correspondence Tanvi P. Vaidya, 3, Krishnatara, Sindh \\ Society, Baner road, Aundh, Pune 411007, Maharashtra, India \\ (e-mail: tanvivaidya5@gmail.com).
}

\begin{abstract} Keywords

- carcinoma

- male breast

- mammography

- ultrasonography

The male breast can be afflicted with a wide spectrum of benign and malignant masses, similar to the female breast. A systematic radiological evaluation using mammography, ultrasonography, and when appropriate, magnetic resonance imaging, could aid this differentiation and provide clues to the diagnosis. In this article, we present six cases of male breast masses with an emphasis on the role of imaging in characterization and diagnosis.
\end{abstract}

\section{Introduction}

The male breast can be afflicted with a wide spectrum of disease processes, similar to the female breast. ${ }^{1}$ The adult male breast reveals a predominance of skin and subcutaneous fat; atrophic ducts and stromal tissue constitute a minor proportion of the mammary tissue. ${ }^{2}$ A variety of benign and malignant masses can arise from each of these components. Various imaging characteristics of a breast mass, such as its shape, margins, composition, and enhancement characteristics serve as important predictors of malignancy or benignity; thus, a systematic imaging evaluation using mammography, ultrasonography (US), and, occasionally, magnetic resonance imaging (MRI) is of utmost importance to facilitate this distinction and guide clinical decisions. ${ }^{3,4}$ Mammography is the imaging modality for the preliminary evaluation of a mass in the male breast. ${ }^{5}$ Any mammographic abnormality ideally necessitates a targeted US for further characterization.

Based on the clinical and radiologic index of suspicion for malignancy, an image-guided biopsy may be performed for tissue diagnosis. Rarely, further imaging with an MRI breast may be recommended in cases where conventional imaging is equivocal. ${ }^{6}$

In this article, we present six cases of male breast masses with an emphasis on the role of imaging in characterization and diagnosis. The purpose of this article is to understand the role of imaging in the evaluation of male breast masses and to provide an overview of the imaging appearances of certain benign and malignant masses arising in the male breast.

\section{Case 1}

A 35-year-old man with neuroendocrine carcinoma of the ethmoid sinus, previously treated with three cycles of chemotherapy and radiotherapy, came to our institute for further management. An fluorodeoxyglucose (FDG) positron emission tomography-computed tomography (PET-CT) was performed to evaluate disease status; it revealed a metabolically active, diffuse soft tissue mass in the retroareolar region of the left breast (-Fig. 1). No evidence of active disease was seen elsewhere. On clinical examination, there was mild tenderness in this region, with no obvious palpable mass. Mammography revealed an ill-defined subareolar isodensity in the retroareolar region of the left breast, with linear projections in the posterior aspect of the density extending into the adjacent fat ( $\boldsymbol{- F i g . 2 A}$ ). No calcifications were seen within. There was no associated thickening of the skin/nipple-areola complex (NAC). US revealed an irregular, hypoechoic mass with extensions into the surrounding tissue showing no internal vascularity, suggestive of gynecomastia published online July 28, 2021
DOI https://doi.org/

10.1055/s-0041-1734358 ISSN 0971-3026

\section{(C) 2021. Indian Radiological Association.}

This is an open access article published by Thieme under the terms of the Creative Commons Attribution-NonDerivative-NonCommercial-License, permitting copying and reproduction so long as the original work is given appropriate credit. Contents may not be used for commercial purposes, or adapted, remixed, transformed or built upon. (https://creativecommons.org/licenses/by-nc-nd/4.0/).

Thieme Medical and Scientific Publishers Private Ltd. A-12, Second Floor, Sector -2, NOIDA -201301, India 

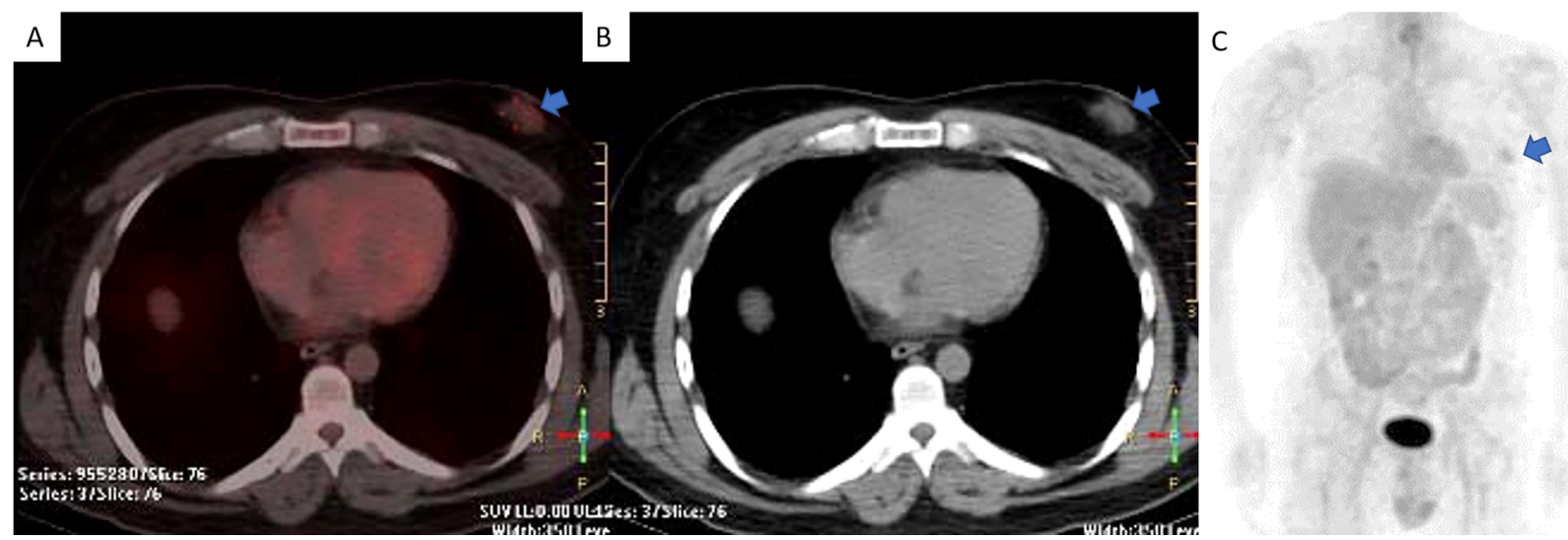

Fig. 1 (A-C) Fluorodeoxyglucose (FDG) positron emission tomography-computed tomography (PET-CT) images showing low-grade FDG uptake in the left breast soft tissue (blue arrow in $\mathbf{A}$ ). The soft tissue is well seen on plain CT images (blue arrow in B). Maximum intensity projection (MIP) images showing mildly FDG avid left breast soft tissue (blue arrow in C).

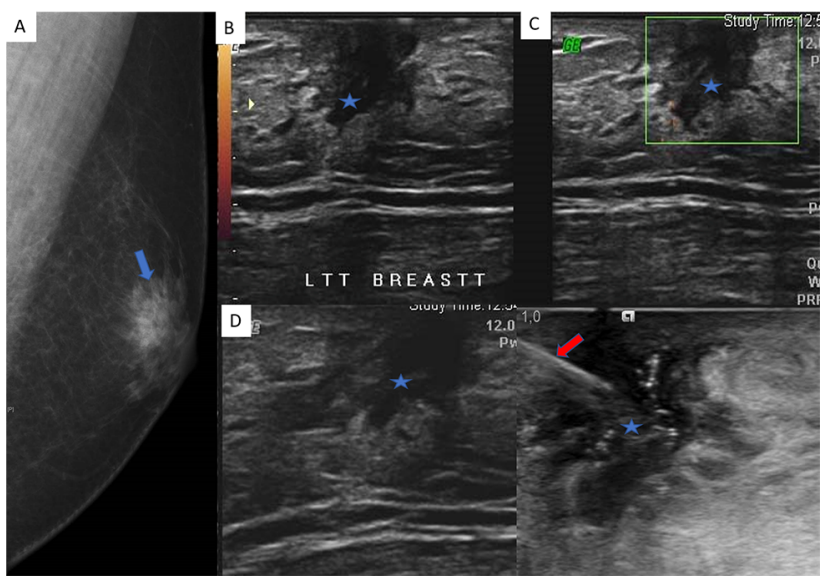

Fig. 2 (A-D) Mammography image of the left breast showing an ill-defined subareolar iosdensity with linear projections in the posterior aspect of the lesion on the mediolateral oblique (MLO) view (blue arrow in A). Ultrasonography (US) images showing a hypoechoic, mass with extensions into the surrounding tissue (blue asterisk in B) and no internal vascularity (blue asterisk in C). A US-guided core biopsy (red arrow in $\mathbf{D}$ indicating the biopsy needle) was performed from the lesion (blue asterisk in $\mathbf{D}$ ), following which the diagnosis of gynecomastia was confirmed.

( - Fig. 2B and C ). A US-guided core biopsy from the lesion ( - Fig. 2D) revealed benign breast parenchyma with no evidence of malignancy. The diagnosis of gynecomastia was confirmed. The patient has been kept on follow-up for the past 6 years and has been disease free.

\section{Case 2}

A 57-year-old male presented with a progressively increasing mass in the right breast associated with occasional blood-stained nipple discharge. On clinical examination, a 5 $\times 4 \mathrm{~cm}$ mass was palpable in the upper outer quadrant with evidence of skin and nipple involvement. Mammography revealed a high-density mass with partially spiculated margins in the outer central region of the right breast associated with thickening and retraction of the overlying skin, NAC ( - Fig. 3A and B ). US revealed an irregular, hypoechoic,

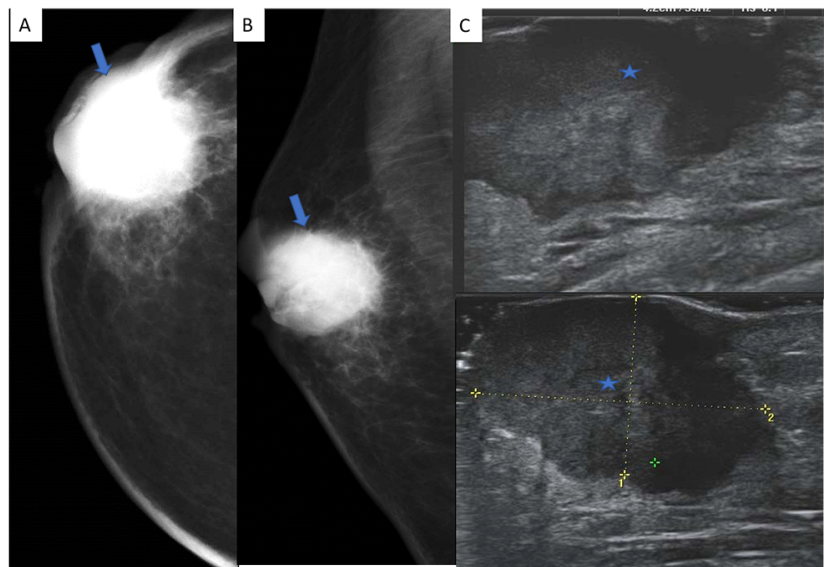

Fig. 3 (A-C) Mammography images of the right breast showing a high density mass with partially spiculated margins in the outer central region associated with thickening and retraction of the skin, nipple-areola complex (NAC) (blue arrow in $\mathbf{A}$ [craniocaudal [CC] view], blue arrow in B [mediolateral oblique [MLO] view]). Ultrasonography (US) images showing an irregular, hypoechoic mass with angular margins (blue asterisks in C) at the 9 o' clock position.

solid mass with angular margins at the 9 o'clock position (-Fig. 3C). US-guided core biopsy revealed invasive ductal carcinoma (IDC) grade III; negative for estrogen receptor (ER), progesterone receptor (PR), and human epidermal growth factor receptor 2 (Her-2/neu) on immunohistochemistry (IHC). The patient underwent a modified radical mastectomy (MRM) for localized disease. However, 3 years later he presented with bilateral neck nodes which revealed uptake on FDG PET-CT scans (-Fig. 4A-C); fine-needle aspiration cytology (FNAC) confirmed metastatic adenocarcinoma. The patient was put on palliative chemotherapy but died after a year.

\section{Case 3}

A 61-year-old man presented with a small, painless lump in the periareolar region of the left breast since 4 months. No history of nipple discharge was present. Clinical examination revealed a $1 \times 1 \mathrm{~cm}$ mobile lump at the 12 o'clock 


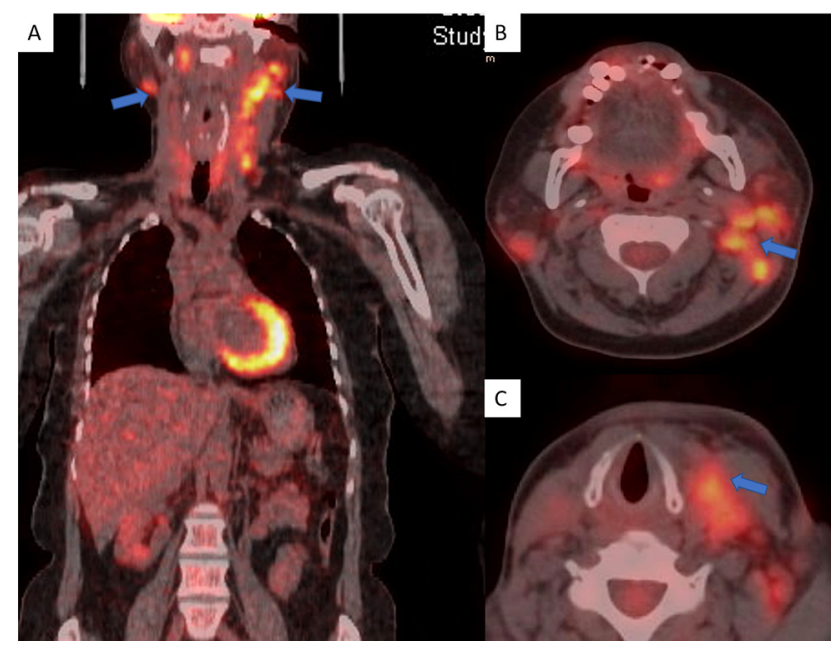

Fig. 4 (A-C) Fluorodeoxyglucose (FDG) positron emission tomography-computed tomography (PET-CT) images post right mastectomy showing FDG avid bilateral cervical lymph nodes (blue arrows in A), left more than right. Axial images demonstrate FDC avid left level II (blue arrow in B) and left level IV lymph nodes (blue arrow in C).

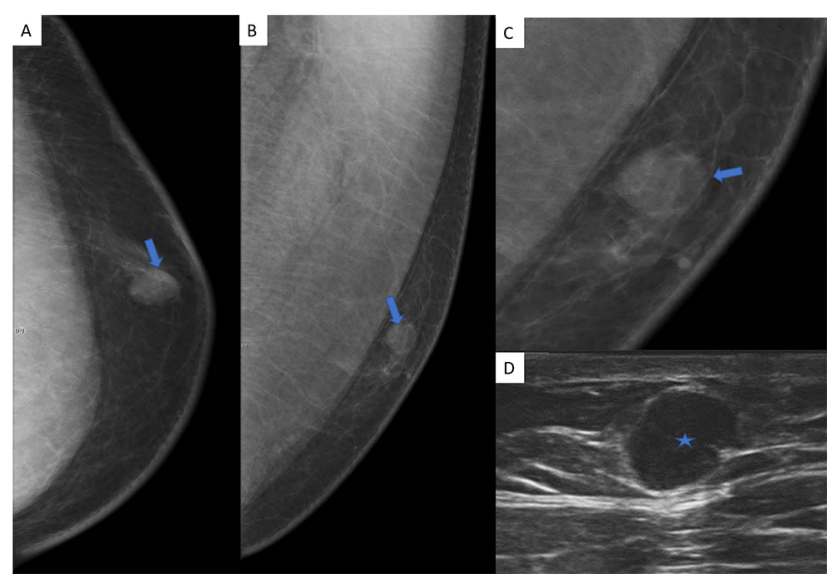

Fig. 5 (A-D) Mammography images of the left breast showing a round, isodense mass with partially circumscribed and partly indistinct margins in the upper central region. No evidence of skin/nipple-areola complex (NAC) thickening or retraction is seen (blue arrow in $\mathbf{A}$ [craniocaudal [CC] view], blue arrows in $\mathbf{B}$ and $\mathbf{C}$ [mediolateral oblique [MLO] view]). Ultrasonography (US) image showing an oval, hypoechoic, wider than taller, solid lesion with relatively circumscribed margins showing a nonparallel orientation and no posterior features (blue asterisk in $\mathbf{D}$ ).

position in the left breast. No axillary nodes were palpable. On mammography, a round, isodense mass with partially circumscribed and partly indistinct margins was seen in the upper central region ( $\mathbf{- F i g}$. 5A-C). No evidence of skin/NAC thickening or retraction was seen. US revealed an oval, hypoechoic, solid lesion with relatively circumscribed margins which appeared wider than taller, showing a nonparallel orientation and no posterior features ( $\boldsymbol{- F i g}$. 5D). The lesion was assigned a category of American College of Radiology (ACR) Breast Imaging-Reporting and Data System (BI-RADS) 4b. The patient underwent an excision biopsy which revealed infiltrating papillary carcinoma, grade II with apocrine change. IHC revealed positivity for ER (90\%), PR (30\%), and was negative for Her-2/neu. The patient underwent a left MRM and has been disease free since then.

\section{Case 4}

A 71-year-old male presented with a painless lump in the right breast since 1 year, gradually increasing in size. There was no history of trauma to the chest wall and no nipple discharge. On examination, a relatively well-defined mass was palpable in the upper central region of the right breast. There was no evidence of skin thickening, nipple retraction, or enlarged axillary nodes. Mammography revealed a mixed density mass with circumscribed margins in the upper central region of the right breast ( - Fig. $\mathbf{6 A}$ and $\mathbf{B}$ ). US revealed an iso- to hyperechoic mass with circumscribed margins at the 2 o'clock position ( - Fig. $6 \mathbf{6 C}$ ). FNAC of the mass revealed the presence of spindle cells, raising the possibility of a sarcoma. This was followed by an MRI, which revealed a mass in the right breast, appearing predominantly isointense to fat on T1- and T2-weighted images with hypointense nodules within ( - Fig. $7 \mathbf{A}$ and $\mathbf{B}$ ). Postcontrast images revealed an irregular, heterogeneously enhancing mass with few enhancing incomplete septae and solid nodules, showing type-3 kinetics. These features were highly suggestive of a liposarcoma ( - Fig. 7C). A plain CT of the thorax ( - Fig. 7D) was done to look for lung metastases, which was unremarkable. The patient underwent a radical mastectomy. Histopathology revealed a liposarcoma with an inflammatory myofibroblastic element. On IHC, the spindle cell component was positive for CDK-4, S-100, smooth muscle actin, CD-34, and desmin.

\section{Case 5}

A 60-year-old man presented with a painless, palpable lump in the retroareolar region of the left breast since 3 months. It was associated with scanty, blood-stained nipple discharge. There were no other pertinent features in his medical history.

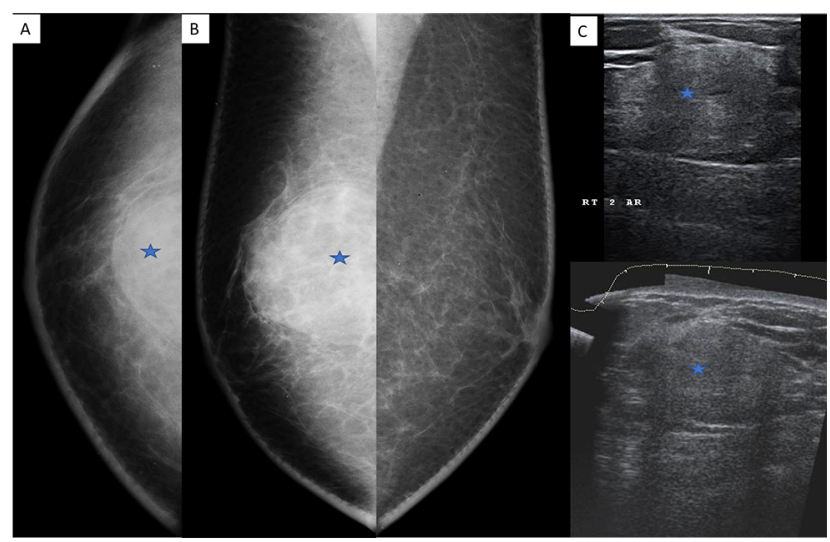

Fig. 6 (A-C) Mammography images showing a mixed density mass with circumscribed margins in the upper central region of the right breast (blue asterisk in $\mathbf{A}$ [craniocaudal [CC] view], blue asterisk in B [mediolateral oblique [MLO] view]). Ultrasonography (US) image showing an iso- to hyperechoic mass with circumscribed margins at the 2 o' clock position (blue asterisk in C). 


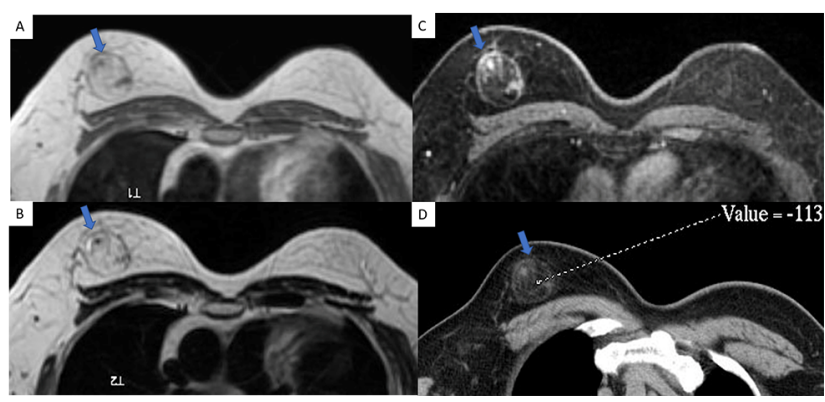

Fig. 7 (A-D) Axial magnetic resonance imaging (MRI) images revealed a mass in the upper central region of the right breast (blue arrows in $\mathbf{A}, \mathbf{B})$, with predominant isointensity to fat on $\mathrm{T1}$ - (A) and T2-weighted (B) images and hypointense solid nodules within. Contrast-enhanced MRI image showing an irregular, heterogeneously enhancing mass with few enhancing incomplete septae and solid nodules (blue arrow in C). Plain computed tomography (CT) of the thorax demonstrating fat content within the mass (blue arrow in $\mathbf{D}$ ).

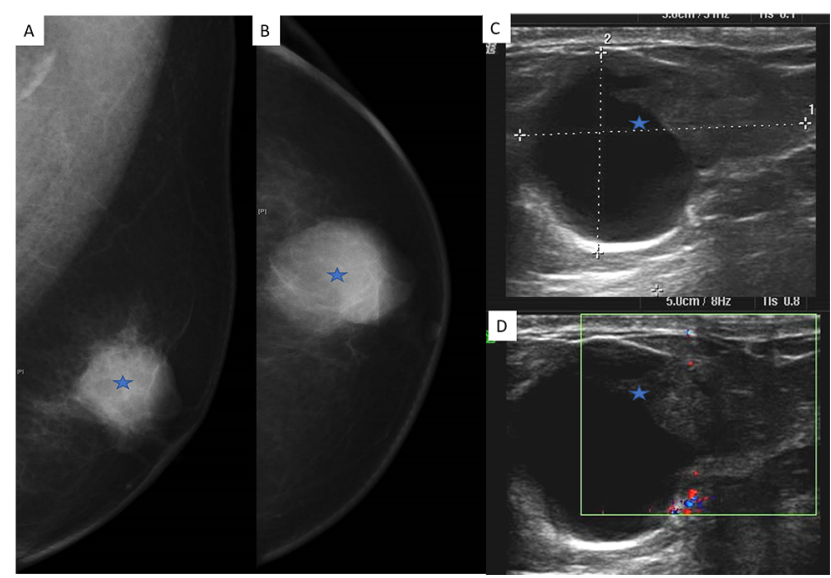

Fig. 8 (A-D) Mammography images of the left breast showing a high-density, oval mass with areas of low density within, gentle lobulations, and circumscribed margins in the retroareolar location. No calcifications or architectural distortion was seen (blue asterisk in A [mediolateral oblique [MLO] view], blue asterisk in B [craniocaudal [CC] view]). Ultrasonography (US) image showing a complex solid-cystic, irregular lesion (blue asterisks in C, D) with an iso- to hypoechoic focal solid component along the lateral cyst wall. It revealed no significant internal vascularity (D).

There was no history of local trauma. Clinical examination revealed a rounded, palpable mass in the subareolar region of the left breast. There were no palpable axillary lymph nodes, no nipple retraction. Mammography revealed a high-density oval mass with areas of low density within, with gentle lobulations and circumscribed margins in the retroareolar location ( - Fig. 8A and $\mathbf{B}$ ). No calcifications or architectural distortion were seen. US revealed a $1.9 \times 2.3 \mathrm{~cm}$, complex, solid-cystic, irregular lesion with an iso- to hypoechoic focal solid component along the lateral cyst wall ( - Fig. $\mathbf{8 C}$ ). It revealed no significant internal vascularity; mild peripheral vascularity was noted ( $\boldsymbol{- F i g}$. 8D). There were no suspicious axillary lymph nodes. We classified the lesion as ACR BI-RADS category $4 \mathrm{~b}$ (intermediate suspicion for malignancy). An excision biopsy was performed which revealed a benign intraductal papilloma with foamy histiocytic reaction and no evidence of atypia or malignancy.

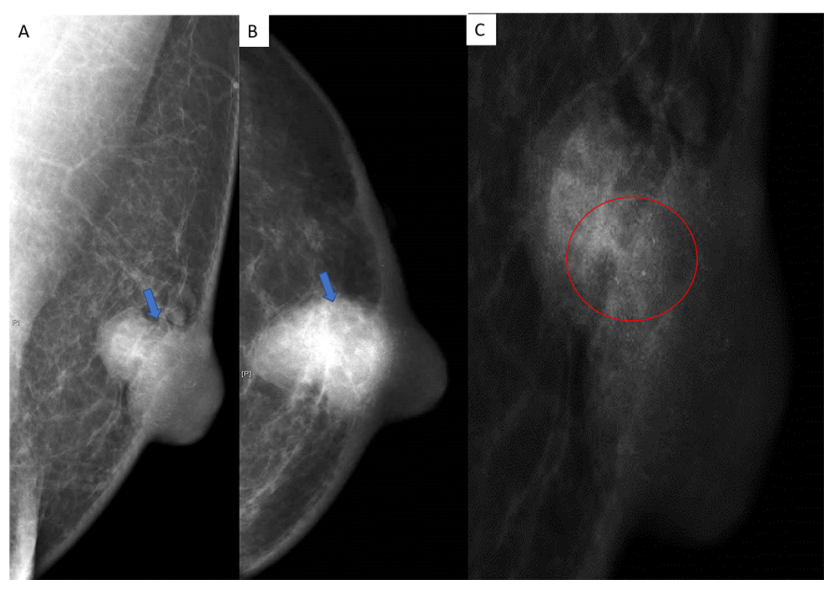

Fig. 9 (A-C) Mammography images showing an oval, isodense mass in the retroareolar region of the left breast, with well-circumscribed margins. There is associated thickening of the skin and nipple-areola complex (NAC); no nipple retraction was seen (blue arrow in $\mathbf{A}$ [mediolateral oblique [MLO] view], blue arrow in B [craniocaudal [CC] view]). Few monomorphic microcalcifications are seen within (red circle in C).

\section{Case 6}

A 49-year-old male presented with a palpable, mildly painful swelling in the left breast, progressively increasing over a period of 4 months. On clinical examination, a small, firm mass was palpable in the inner central region, adjacent to the NAC. No palpable axillary lymph nodes were present. Mammography revealed an oval, isodense mass in the retroareolar region of the left breast, with circumscribed margins and few monomorphic microcalcifications within. There was associated thickening of the skin and NAC; however, no

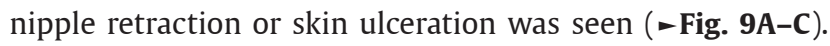
The lesion was assigned a category of ACR BI-RADS 4a, and the patient underwent a core biopsy. Histopathologic examination revealed a pilomatrixoma. He underwent an NAC sparing excision of the lesion and has been disease-free since.

\section{Discussion}

Gynecomastia is the most common benign pathology of the male breast, believed to arise secondary to elevated estrogen levels. ${ }^{7}$ The diagnosis of gynecomastia is generally made based on a history of mild breast pain and clinical examination, which reveals a soft subareolar mass or diffuse breast enlargement. ${ }^{1}$ We encountered an unusual case of unilateral gynecomastia, which was detected incidentally on a PET-CT study performed in a treated case of sinonasal carcinoma. A few authors have reported gynecomastia as a possible cause of false-positive imaging on FDG PET-CT studies, which may be considered in cases presenting with FDG uptake in the breast. ${ }^{89}$ However, given the presence of a soft tissue mass in the breast on CT images coupled with tracer uptake, the possibility of a metastatic deposit or a second primary needed to be excluded. This was done using mammography, US, and ultimately a biopsy. The mammography and US evaluation revealed imaging features suggestive of dendritic gynecomastia, characterized by finger-like extensions 
radiating outwards from a subareolar density on mammography and a hypoechoic, subareolar soft tissue on US, respectively. ${ }^{1}$ Although these imaging features were highly suggestive of the diagnosis in our case, a biopsy confirmation was necessary to establish the diagnosis in this setting.

Male breast cancer is an infrequently encountered disease, accounting for only $0.7 \%$ of all breast cancers. ${ }^{2}$ Of all the histologic types of male breast cancers, IDC is the most common variant constituting $85 \%$ of all cases. ${ }^{1,2}$ We encountered a case of IDC with the classical imaging features of malignancy, which is the most frequently encountered presentation of this subtype. ${ }^{2}$ The other case of breast carcinoma in our series was an invasive intraductal papillary carcinoma, the second most common subtype of male breast cancer. ${ }^{2,10}$ It differed significantly from IDC in its imaging features, as it had only mildly suspicious characteristics on mammography. Similar findings have been reported by a few authors ${ }^{10,11}$; thus, even a new-onset, radiologically benign-appearing mass in an elderly male must be viewed with caution and a papillary carcinoma must be excluded by means of a biopsy.

A vast majority of male breast carcinomas are strongly positive for ER and PR on IHC ${ }^{12}$; however, we encountered an unusual case of triple-negative breast cancer (TNBC). To the best of our knowledge, only one other such case has been reported in the literature in the context of male breast cancer. ${ }^{13}$ The mammography and US findings in this case were similar to those described by us. Researchers have observed that the prognosis of male TNBCs is significantly worse than that of female TNBCs, with a greater risk of recurrence and mortality. ${ }^{14,15}$ Our patient presented with distant metastases to the cervical lymph nodes, and no deposits to the usual sites of distant metastases, that is, the bones, liver, and lungs. ${ }^{16}$ No disease recurrence was seen locally. Based on our experience, we recommend close, follow-up evaluation for patients with TNBC, as this may aid early detection of recurrence.

Primary malignant tumors originating from the mesenchymal tissue of the breast are extremely rare accounting for $<1 \%$ of all breast cancers, ${ }^{17}$ of which liposarcomas constitute only approximately $0.3 \% .^{18}$ Very few case reports of this rare entity are available in literature. Mammography findings may reveal a mixed density, lobulated mass with areas of lower density corresponding to the fat component. ${ }^{19} \mathrm{We}$ did encounter lucent areas on mammography; however, the US findings were nonspecific. The fat components were well seen on MRI and CT images, which enabled us to arrive at the diagnosis on imaging. Histopathology and IHC confirmed the diagnosis; the positivity of tumor cells for CDK-4 was of particular importance for the diagnosis of a well-differentiated liposarcoma. ${ }^{20}$ Although tissue diagnosis is mandatory, we observed that MRI certainly adds value in the evaluation of suspected breast sarcomas, as a diagnostic tool as well as for disease staging to plan further management.

We encountered a rare case of benign intraductal papilloma of the left breast, causing single duct, blood-stained discharge. The imaging and clinical examination findings were suspicious for malignancy, thus necessitated a tissue diagnosis. Surgical excision was preferred to a percutaneous biopsy to obtain an accurate diagnosis of the complex solid-cystic lesion seen on US, as it may be difficult to precisely target and sample the solid component via US-guided biopsy after decompression of the cyst. Due to the overlapping imaging findings of benign and malignant papillary lesions, ${ }^{21,22}$ a definite differentiation is often not possible through imaging alone, as seen in our case. A possible imaging feature favoring benignity could be the retroareolar location of the lesion since malignant intraductal papillomas tend to be more peripherally located. ${ }^{23}$ However, considering the other parameters in our case, such as patient age more than 50 years and lesion size more than $1.5 \mathrm{~cm}$, the likelihood of malignancy was considerably higher. ${ }^{24}$ Given their rarity of occurrence in practice, these predictive features have not yet been established in the context of male breast papillomas. We believe that more case reports like ours could be compiled and collectively analyzed for a better understanding of this rare entity in future.

Pilomatrixoma of the breast is exceedingly uncommon, with very few cases reported in literature. ${ }^{25}$ Originally termed as a calcifying epithelioma of sebaceous gland, these lesions are more common in the first two decades with a predominance in females. ${ }^{26}$ These lesions are essentially benign dermatological tumors arising from the matrix cells of hair follicles, possibly due to inflammatory response to prior skin trauma. ${ }^{27} \mathrm{~A}$ few investigators have described the mammographic features of a pilomatrixoma in the male breast, appearing as an oval-shaped, circumscribed mass with pleomorphic calcifications, thereby mimicking carcinoma. ${ }^{26,28} \mathrm{We}$ encountered a similar morphology mass in our case, except that the calcifications were monomorphic, favoring a benign etiology. Pilomatrixomas have been reported to show benign features on US, usually appearing iso- to hypoechoic, well-circumscribed masses with a parallel orientation. ${ }^{29}$ The imaging appearances are not very specific; thus, the imaging differentials may include benign conditions such as epidermal inclusion cysts, fibrocystic changes, papillomas, as well as malignant neoplasms such as invasive papillary carcinoma. ${ }^{26}$ Nevertheless, it is important for the radiologist to be aware of this entity so that the imaging features can be correlated with histopathological analysis, to ultimately decide the need for surgical excision. Pilomatrixoma of the male breast though uncommon should be considered in the differential diagnosis of a male breast mass with predominantly benign features on imaging. ${ }^{26}$

\section{Conclusion}

To conclude, a variety of benign and malignant masses can arise in the male breast. In recent years, there has been an increase in the number of diagnostic scans performed in men presenting with breast-related complaints, such as breast pain, nipple discharge, breast lumps to name a few, owing to improved awareness and a better understanding of pathologies. Thus, a familiarity with the imaging features of certain common as well as uncommon, benign and malignant entities will facilitate lesion characterization and add value to the radiology report. Judicious use of mammography, US, and when appropriate, MRI in the evaluation of male breast 
masses will enable the radiologist to arrive at a diagnosis and guide further management.

\section{Key Messages}

A variety of benign and malignant masses can occur in the male breast.

These masses have characteristic imaging features that can be evaluated using mammography, ultrasonography (US), and magnetic resonance imaging as required.

Familiarity with these imaging features will facilitate lesion characterization and aid diagnosis.

\section{Declaration of Patient Consent}

The authors certify that they have obtained all appropriate patient consent forms. In the form the patient(s) has/have given his/her/their consent for his/her/their images and other clinical information to be reported in the journal. The patients understand that their names and initials will not be published and due efforts will be made to conceal their identity, but anonymity cannot be guaranteed.

\section{Financial Support and Sponsorship}

None.

\section{Conflict of Interest}

There are no conflicts of interest.

\section{Acknowledgments}

None.

\section{References}

1 Nguyen C, Kettler MD, Swirsky ME, et al. Male breast disease: pictorial review with radiologic-pathologic correlation. Radiographics 2013;33(3):763-779

2 Iuanow E, Kettler M, Slanetz PJ. Spectrum of disease in the male breast. AJR Am J Roentgenol 2011;196(3):W247-59

3 Lazarus E, Mainiero MB, Schepps B, Koelliker SL, Livingston LS. BI-RADS lexicon for US and mammography: interobserver variability and positive predictive value. Radiology 2006;239(2):385-391

4 Liberman L, Morris EA, Lee MJ, et al. Breast lesions detected on MR imaging: features and positive predictive value. AJR Am J Roentgenol 2002;179(1):171-178

5 Patterson SK, Helvie MA, Aziz K, Nees AV. Outcome of men presenting with clinical breast problems: the role of mammography and ultrasound. Breast J 2006;12(5):418-423

6 Shaw A, Smith B, Howlett D. Male breast carcinoma and the use of MRI. Radiol Case Rep 2015;6(3):455

7 Chau A, Jafarian N, Rosa M. Male breast: clinical and imaging evaluations of benign and malignant entities with histologic correlation. Am J Med 2016;129(8):776-791

8 Wang HY, Jeng LB, Lin MC, Chao CH, Lin WY, Kao CH. 18F-FDG $\mathrm{PET} / \mathrm{CT}$ in detection of gynecomastia in patients with hepatocellular carcinoma. Clin Imaging 2013;37(5):942-946

9 Ramtahalsing $\mathrm{R}$, Arens AI, Vliegen RF, Teule GJ, van den Ende PL, Beets-Tan RG. False positive 18F-FDG
PET/CT due to gynaecomastia. Eur J Nucl Med Mol Imaging 2007;34(4):614

10 Yee C, Wan BA, Drost L, et al. Papillary carcinoma of the male breast: a case series. Ann Breast Cancer Ther 2017;1:24-27

11 Arora R, Gupta R, Sharma A, Dinda AK. Invasive papillary carcinoma of male breast. Indian JPathol Microbiol2010;53:135-137

12 Rayson D, Erlichman C, Suman VJ, et al. Molecular markers in male breast carcinoma. Cancer 1998;83(9):1947-1955

13 Zhang L, Zhang C, Yang Z, et al. Male occult triple-negative breast cancer with dermatomyositis: a case report and review of the literature. OncoTargets Ther 2017;10:5459-5462

14 Montagna E, Bagnardi V, Rotmensz N, et al. Immunohistochemically defined subtypes and outcome in occult breast carcinoma with axillary presentation. Breast Cancer Res Treat 2011;129(3):867-875

15 Arslan UY, Oksüzoğlu B, Ozdemir N, et al. Outcome of non-metastatic male breast cancer: 118 patients. Med Oncol 2012;29(2):554-560

16 Agrawal S, Jayant K, Agarwal RK, Dayama KG, Arora S. An unusual case of metastatic male breast cancer to the nasopharynx-review of literature. Ann Palliat Med 2015;4(4):233-238

17 Sinn HP, Kreipe H. A Brief Overview of the WHO Classification of Breast Tumors, 4th Edition, Focusing on Issues and Updates from the 3rd Edition. Breast Care (Basel 2013;8(2):149-154

18 Demaria S, Yee HT, Cangiarella J, Cohen JM, Chhieng DC. Fine needle aspiration of primary pleomorphic liposarcoma of the breast. A case report. Acta Cytol 1999;43(6):1131-1136

19 Briski LM, Jorns JM. Primary breast atypical lipomatous tumor/ well-differentiated liposarcoma and dedifferentiated liposarcoma. Arch Pathol Lab Med 2018;142(2):268-274

20 Binh MB, Sastre-Garau X, Guillou L, et al. MDM2 and CDK4 immunostainings are useful adjuncts in diagnosing well-differentiated and dedifferentiated liposarcoma subtypes: a comparative analysis of 559 soft tissue neoplasms with genetic data. Am J Surg Pathol 2005;29(10):1340-1347

21 Al Sarakbi W, Worku D, Escobar PF, Mokbel K. Breast papillomas: current management with a focus on a new diagnostic and therapeutic modality. Int Semin Surg Oncol 2006;3:1

22 Lam WW, Chu WC, Tang AP, Tse G, Ma TK. Role of radiologic features in the management of papillary lesions of the breast. AJR Am J Roentgenol 2006;186(5):1322-1327

23 Kil WH, Cho EY, Kim JH, Nam SJ, Yang JH. Is surgical excision necessary in benign papillary lesions initially diagnosed at core biopsy? Breast 2008;17(3):258-262

24 Youk JH, Kim EK, Kwak JY, Son EJ, Park BW, Kim SI. Benign papilloma without atypia diagnosed at US-guided 14-gauge core-needle biopsy: clinical and US features predictive of upgrade to malignancy. Radiology 2011;258(1):81-88

25 Hubeny CM, Sykes JB, O'Connell A, Dogra VS. Pilomatrixoma of the adult male breast: a rare tumor with typical ultrasound features. J Clin Imaging Sci 2011;1:12

26 Clark A, Leddy R, Spruill L, Cluver A. Pilomatrixoma, a rare mimicker of male breast cancer. J Clin Imaging Sci 2019;9:46

27 Nori J, Abdulcadir D, Giannotti E, Calabrese M. Pilomatrixoma of the breast, a rare lesion simulating breast cancer: a case report. J Radiol Case Rep 2013;7(10):43-50

28 Becker TS, Moreira MA, Lima LA, de Oliveira EL, Freitas-Júnior R. Pilomatrixoma mimicking breast cancer in man. Breast J 2010;16(1):89-91

29 Kapoor A, Narayanan R, Tandon A, Santosh AK. Pilomatricoma: an unusual cause of lump in a male breast. J Clin Ultrasound 2018;46(3):209-211 